\title{
Correlation between Serum Uric Acid and HbA1c Levels in Patients with Type 2 Diabetes Mellitus
}

\author{
Rifan Nugraha, ${ }^{1}$ Nina Tristina, ${ }^{2}$ Miftahurachman ${ }^{3}$ \\ ${ }^{1}$ Faculty of Medicine Universitas Padjadjaran, Indonesia, ${ }^{2}$ Department Clinical Pathology Faculty \\ of Medicine Universitas Padjadjaran/Dr. Hasan Sadikin General Hospital Bandung, Indonesia, \\ ${ }^{3}$ Departement of Internal Medicine Faculty of Medicine Universitas Padjadjaran/Dr. Hasan \\ Sadikin General Hospital Bandung, Indonesia
}

\begin{abstract}
Background: Indonesia ranks fourth among countries with the highest number of diabetics. Increasing evidence suggests that hyperuricemia is an independent risk factor for impaired fasting glucose (IFG) and type 2 diabetes. Some observational studies have identified elevated uric acid concentration as a risk factor for diabetes, while others have found an inverse relationship. The present study was conducted to discover the strength and the direction of the correlation.

Methods: This study was an analytical cross-sectional study conducted from May to October 2014. The study subjects were 56 medical records of new outpatients diagnosed with T2DM in the Internal Medicine Polyclinic who underwent laboratory examination at the Clinical Pathology Unit of Dr. Hasan Sadikin General Hospital, January-December 2013.

Result: The correlation between HbA1c and uric acid levels on the whole subject revealed a weak but significant negative correlation $(\mathrm{r}=-0.354, \mathrm{p}=0.007)$. There was an insignificant negative correlation in male subjects $(\mathrm{r}=-0.405, \mathrm{p}=0.120)$; in female subjects $(\mathrm{r}=-0.319, \mathrm{p}=0.05)$, the correlation was a weak but insignificant negative correlation.

Conclusions: There is a weak correlation between HbA1c and uric acid levels in patients with Diabetes Mellitus type 2 .
\end{abstract}

Keywords: Correlation, HbA1c, T2DM, uric acid

\section{Introduction}

In 2010, Indonesia ranks fourth among countries with the highest number of diabetics with estimated 8.4 million people, and these numbers are subject to increase in 2030 to a staggering 21.3 million people. ${ }^{1}$ Type 2 Diabetes Mellitus (T2DM) is characterized by chronic hyperglycemia that causes the formation of early product of glycosylation, to which glycated hemoglobin (HbA1c) is an analog. HbA1c is a marker that reflects average plasma glucose over the previous 2-3 months. Complications from chronic hyperglycemia manifest as microangiopathy and macroangiopathy, such as coronary artery disease, cerebral vascular disease, and peripheral artery disease. Uric acid is the endproduct of purine metabolism. A previous study by Ogbera et al. in 2010 reveals that the prevalence of hyperuricemia, defined as serum uric acid is higher than $7 \mathrm{mg} / \mathrm{dL}$ for men and $5.7 \mathrm{mg} / \mathrm{dl}$ for women, in T2DM patients is $25 \% .{ }^{2} \mathrm{HbA} 1 \mathrm{c}$ levels correlate with serum uric acid levels through competing mechanism in uric acid resorption in kidney, mediated by glycosuric effect on uricosuria. ${ }^{3}$

Studies exploring the correlation between HbA1c and serum uric acid levels are relatively scarce compared to the extensive research aiming to analyze the correlation between blood glucose and serum uric acid levels. ${ }^{3,4}$ The correlation between HbA1c and serum uric acid levels was first studied by Choi et al. in 2008, with the result showing a bell-shaped relation. ${ }^{5}$ It is crucial that this correlation is completely understood so that the clinician may predict when T2DM patients are at risk of hyperuricemia. A conflicting result is found by another study which states, uric acid concentration is emerging as a potential marker of diabetes risk. ${ }^{6}$

Based on this background, this study

Correspondence: Rifan Nugraha, Faculty of Medicine Universitas Padjadjaran, Jalan Raya Bandung-Sumedang Km. 21, Jatinangor, Jawa Barat, Indonesia, Email: rifan.nugraha@gmail.com 
explored the correlation between HbA1c and serum uric acid levels in new outpatients diagnosed with T2DM in the Internal Medicine Polyclinic who underwent laboratory examination at the Clinical Pathology Unit, Dr. Hasan Sadikin General Hospital from January to-December 2013 and was conducted to discover the strength and the direction of the correlation.

\section{Methods}

This study was a retrospective study conducted in an analytic cross-sectional method during the period May-October 2014. The subjects were new outpatients diagnosed with T2DM in the Internal Medicine Polyclinic who underwent laboratory examination at the Clinical Pathology Unit, Dr. Hasan Sadikin General Hospital from January to December 2013. Materials used in this study were medical records which met the inclusion criteria that were subjects with complete data of fasting blood glucose levels (mg/dL), 2-hour postprandial glucose levels (mg/dL), HbA1c levels (\%), and serum uric acid levels (mg/dL).
The sampling method used was consecutive sampling with a minimum of 30 samples. This study was approved by the Ethical Clearance Committee of Dr. Hasan Sadikin General Hospital.

The data collection process from medical records resulted in 1,275 outpatients diagnosed with T2DM at the Internal Medicine Polyclinic, Dr. Hasan Sadikin General Hospital. Out of these 1,275 outpatients, 231 were new in the period of January-December 2013. After consecutive sampling was carried out, the subjects who met the inclusion criteria were 56 patients. Normality test was conducted on continuous variables, including continuous variables in baseline characteristics, fasting blood glucose levels, 2-hour postprandial glucose levels, HbA1c levels, and serum uric acid levels. The variables were then foremost presented in a descriptive analysis. The statistical analysis in this study was correlation analysis with two continuous variables. If the serum uric acid and $\mathrm{HbA} 1 \mathrm{c}$ levels were normally distributed, the statistic test used would be Pearson correlation coefficient; otherwise, Spearman's rho correlation coefficient would be used.

Table 1 The clinical Characteristic of Typ 2 Diabetes Mellitus Patients

\begin{tabular}{|c|c|c|c|}
\hline Characteristic & Men & Women & Total \\
\hline Sex & $16(28.57 \%)$ & $40(71.43 \%)$ & $56(100 \%)$ \\
\hline Age, years & $57 \pm 11.1^{*}$ & $57 \pm 7.2^{*}$ & $56.7 \pm 8.1^{*}$ \\
\hline \multicolumn{4}{|l|}{ Lipid Profile } \\
\hline Total cholesterol, mg/dl & $202 \pm 52.7^{*}$ & $205 \pm 44.5^{*}$ & $204 \pm 46.6^{*}$ \\
\hline $\mathrm{HDL}, \mathrm{mg} / \mathrm{dl}$ & $40 \pm 6.7^{*}$ & $50 \pm 9.5^{*}$ & $47 \pm 10^{*}$ \\
\hline $\mathrm{LDL}, \mathrm{mg} / \mathrm{dl}$ & $132 \pm 46.6^{*}$ & $130 \pm 43.2^{*}$ & $130.5^{* *}$ \\
\hline Triglyceride, mg/dl & $180 \pm 84.8^{*}$ & $143^{* *}$ & $149.5^{* *}$ \\
\hline SGOT, u/l & $19^{* *}$ & $15.4 \pm 4.3^{*}$ & $18^{* *}$ \\
\hline SGPT, u/l & $19^{* *}$ & $15 \pm 6^{*}$ & $18^{* *}$ \\
\hline Ureum, mg/dl & $27^{* *}$ & $22^{* *}$ & $23^{* *}$ \\
\hline Creatinine, mg/dl & $1.2^{* *}$ & $0.7^{* *}$ & $0.7^{* *}$ \\
\hline \multicolumn{4}{|l|}{ Proteinuria } \\
\hline$-(\%)$ & $2(15.4 \%)$ & $23(63.9 \%)$ & $25(51 \%)$ \\
\hline$+/-(\%)$ & $1(7.7 \%)$ & $3(8.3 \%)$ & $4(8.2 \%)$ \\
\hline $1+(\%)$ & $5(38.5 \%)$ & $6(16.7 \%)$ & $11(22.45 \%)$ \\
\hline $2+(\%)$ & $1(7.7 \%)$ & $1(2.8 \%)$ & $2(4.2 \%)$ \\
\hline $3+(\%)$ & $4(30.8 \%)$ & $2(5.6 \%)$ & $6(12.24 \%)$ \\
\hline $4+(\%)$ & - & $1(2.8 \%)$ & $1(2 \%)$ \\
\hline
\end{tabular}

Note: ${ }^{*}$ The data were normally distributed and presented in mean \pm standard deviation

** The data were not normally distributed and presented in median. 
Table 2 Laboratory Test Results of Fasting Blood Glucose Levels, 2-Hour Postprandial Glucose Levels, HbA1c Levels, and Serum Uric Acid Levels

\begin{tabular}{lccc}
\hline \multicolumn{1}{c}{ Laboratory Test } & Men & Women & Whole Subject \\
\hline Fasting Blood Glucose (mg/dL) & $167(101-364)$ & $156(94-430)$ & $158(94-430)$ \\
$\begin{array}{l}\text { 2-Hour Postprandial Glucose } \\
\text { (mg/dL) }\end{array}$ & $270(137-590)$ & $230(101-539)$ & $246(101-590)$ \\
HbA1c (\%) & & & \\
Serum Uric Acid (mg/dL) & $8.8(5.3-14.5)$ & $8.6(6.1-37.3)$ & $8.65(5.3-37.3)$ \\
\hline Note: The das & $5.95(3.6-11.5)$ & $5.45(2.8-12.7)$ & $5.65(2.8-12.7)$ \\
\hline
\end{tabular}

Note: The data were not normally distributed, and presented in median and range

\section{Results}

This correlation study was divided between male and female due to the previous study found significant interactions affecting the correlation by sex.5. The correlation between HbA1c and serum uric acid levels in the whole subject was revealed to be a weak negative correlation but significant $(\mathrm{r}=-0.354$, $p=0.007)$. There was a negative correlation but insignificant in male $(r=-0.405, p=0.120)$ and in female, the correlation was weak negative and insignificant $(r=-0.319, p=0.05)$.

\section{Discussion}

In developing countries, the majority of diabetes patients are in the 45-64 age group.1 In this study, the mean \pm standard deviation of ages on whole subject was $56.7 \pm 8.1$ yearold, corresponding to the aforementioned age group. Hyperglycemia in T2DM is too often asymptomatic, hence when the patients are diagnosed, especially in the age above 20 -year-old, the patients have already suffered from complications. ${ }^{7}$ The effect of chronic complication, such as diabetic nephropathy, in this study might become one of the confounding factors to the result of the study.

The correlation between HbA1c and serum uric acid levels on the whole subject showed a weak negative correlation but significant between both variables $(r=-0.354, p=0.007)$, which can be interpreted as the HbA1c levels increased, then the serum uric acid levels decreased. The result of the study conducted by Choi et al. ${ }^{5}$ in 2008, reveals a bell-shaped correlation; the serum uric acid levels increase along the increase of HbA1c until the HbA1c levels reach the interval $6-6.9 \%$, further an increase in $\mathrm{HbA1c}$ results in the decrease of serum uric acid levels. The median of HbA1c levels on the whole subjects in this study was 8.65\%; this value falls on the descending slope of the bell-shaped correlation curve of Choi's. ${ }^{5}$

This negative correlation may appear to be contra-intuitive compared to other studies that find the increase in serum uric acid levels on the hyperinsulinemic state which corresponds to the disturbance of uric acid excretion by kidney is due to the effect of insulin on the uric acid transporters, thus establishing a positive correlation between insulin and uric acid.8 However, Li et al. ${ }^{9}$, in 2011, discovers that the T2DM patient with higher HbA1c levels and longer duration of diabetes are more often associated with the worsening function of pancreatic $\beta$-cell to secrete adequate insulin, thus the correlation between $\mathrm{HbA1c}$ and uric acid on the study shows a negative correlation. ${ }^{9}$

The mechanism responsible for the negative correlation in this study is associated with the mechanism of how the metabolism of glucose influences the metabolism of uric acid; given that $\mathrm{HbA} 1 \mathrm{c}$ levels reflect control value of individual blood glucose levels better. When the glucose levels overcome the threshold of

Table 2 Laboratory Test Results of Fasting Blood Glucose Levels, 2-Hour Postprandial Glucose Levels, HbA1c Levels, and Serum Uric Acid Levels

\begin{tabular}{lcc}
\hline \multicolumn{1}{c}{ Subjects of the Study } & $\begin{array}{c}\text { Spearman's Correlation } \\
\text { Coefficient (r) }\end{array}$ & Significance (p) \\
\hline Whole Subject & -0.354 & $0.007^{* *}$ \\
Male Subjects & -0.405 & 0.120 \\
Female Subjects & -0.319 & 0.05 \\
\hline Note: ${ }^{* *}$ Correlation was significant on 0.05 level
\end{tabular}

Note: ${ }^{* *}$ Correlation was significant on 0.05 level 
kidney for reabsorption, the glucose then starts to be excreted in urine (glycosuria). At this point, the kidney reaches transport maximum, a condition where all of the nephrons work on their maximal capacity to reabsorb glucose. ${ }^{10}$ One of the transporters that has a role in glucose reabsorption is GLUT9 which is also responsible for reabsorbing the uric acid. ${ }^{11}$ Therefore, this competing mechanism decreases the reabsorption of uric acid, thus the negative correlation and the validation of the adage glycosuria causes uricosuria.

This study has several limitations, they are (1) the method used is cross-sectional study, hence the result yields an association that is unable to explain the causative relationship between HbA1c and uric acid levels; (2) confounding factors such as smoking status; BMI; on-going diuretic medication; hypertension; glomerular filtration rate; and protein intake are not pursued. ${ }^{5}$

From this study, we may conclude that there is a weak negative correlation between $\mathrm{HbA} 1 \mathrm{c}$ and serum uric acid levels in new outpatients diagnosed with T2DM in the Internal Medicine Polyclinic who underwent laboratory examination at the Clinical Pathology Unit, Dr. Hasan Sadikin General Hospital from January-December 2013. As the HbA1c levels increase, the serum uric acid levels decrease indicating uricosuric effect on glycosuria, however this correlation is not strong, thus it can be interpreted as there are other factors contributing to the pathophysiologic mechanism of HbA1c and serum uric acid levels. Further studies are suggested to use the prospective method with statistical analysis that may elucidate the causative relationship between $\mathrm{HbA1c}$ and serum uric acid levels in T2DM patients. The prospective method is used on the basis of its capability of analysing the confounding factors that may increase the validity of the study.

\section{References}

1. Wild S, Roglic G, Green A, Sicree R, King H. Global prevalence of diabetes: estimates for the year 2000 and projections for 2030 . Diabetes Care. 2004;27(5):1047-53.

2. Ogbera AO, Azenabor AO. Hyperuricaemia and the metabolic syndrome in type $2 \mathrm{DM}$. Diabetol Metab Syndr. 2010;2:24.

3. Shabana S, Sireesha M, Satyanarayana U. Uric acid in relation to type 2 diabetes mellitus associated with hypertension. Journal of Clinical and Diagnostic Research. 2012;6(7):1140-3.

4. Yuan HJ, Yang XG, Shi XY, Tian R, Zhao ZG. Association of serum uric acid with different levels of glucose and related factors. Chin Med J. 2011;124(10):1443-8.

5. Choi HK, Ford ES. Haemoglobin A1c, fasting glucose, serum C-peptide and insulin resistance in relation to serum uric acid levels - the Third National Health and Nutrition Examination Survey. Rheumatology. 2008;47(5):713-7.

6. Juraschek SP, McAdams-Demarco M, Miller ER. Temporal relationship between uric acid concentration and risk of diabetes in a community-based study population. Am J Epidemiol. 2014;179(6):684-91.

7. Longo DL, Kasper DL, Jameson JL, Fauci AS, Hauser SL, Loscalzo J, editors. Harrison's principles of internal medicine. 18th ed. USA: McGraw-Hill Companies; 2012.

8. Gill A, Kukreja S, Malhotra N, Chhabra N. Correlation of the serum insulin and the serum uric Acid levels with the glycated haemoglobin levels in the patients of type 2 diabetes mellitus. Journal of Clinical and Diagnostic Research. 2013;7(7):1295-7.

9. Li Q, Yang Z, Lu B, Wen J, Ye Z, Chen L, et al. Serum uric acid level and its association with metabolic syndrome and carotid atherosclerosis in patients with type 2 diabetes. Cardiovasc Diabetol. 2011;10:72.

10. Hall JE, Guyton AC, editors. Guyton and Hall textbook of medical physiology. 12th ed. USA: Saunders Elsevier; 2011.

11. Esparza Martin N, Garcia Nieto V. Hypouricemia and tubular transport of uric acid. Nefrologia. 2011;31(1):44-50. 